\title{
E-Health und Zukunft der Informationssysteme im Gesundheitswesen
}

\author{
DOI 10.1007/s11576-012-0347-x
}

\section{Die Autoren}

Prof. Nilmini Wickramasinghe Ph.D. School of Business IT \& Logistics RMIT University Melbourne GPO Box 2476

Melbourne

Victoria 3001

Australien

nilmini.wickramasinghe@rmit.edu.au

Prof. Dr. Stefan Kirn ( $\varangle)$

Institut für Healthcare und Public

Management

Universität Hohenheim

70599 Stuttgart

Deutschland

stefan.kirn@uni-hohenheim.de

Online publiziert: 2013-01-03

This article is also available in English via http://www.springerlink.com and http://www.bise-journal.org: Wickramasinghe N, Kirn S (2012) E-Health and the Future of Healthcare Information Systems. Bus Inf Syst Eng. doi: 10.1007/s12599-012-0245-1.

(C) Springer Fachmedien Wiesbaden 2012
Vor nunmehr 15 Jahren, 1998, wurde mit der Veröffentlichung der „Roland-BergerStudie“ (Roland Berger \& Partner 1998) ein umfassender Prozess zur nachhaltigen Informatisierung des deutschen Gesundheitswesens eingeleitet. Nach einer längeren Konzeptionsphase wurde mit „better IT for health“ (bIT4health) im September 2003 das erste Realisierungsprojekt in Angriff genommen:

„Das Ziel des Projekts „bIT4health“ ist es, die bundesweite Einführung der elektronischen Gesundheitskarte vorzubereiten. Im Mittelpunkt der Arbeiten des Projekts „bIT4health“ steht die Definition einer herstellerneutralen Telematikrahmenarchitektur und Sicherheitsinfrastruktur. Weitere begleitende Aktivitäten sind in den Bereichen Akzeptanzbildung, Projektmanagement, Qualitätssicherung und der wissenschaftlichen Begleitung gebündelt. Das Projektkonsortium „bIT4health“ begleitet die Einführung der elektronischen Gesundheitskarte über die Definitionsphase der Rahmenarchitektur hinaus während der Testphase bis hin zur Einführung und dem ersten Betriebsjahr in 2006“ (ZTG-Telematik-Glossar oJ).

Seither sind rund sieben Jahre vergangen; die ursprünglich zum Januar 2006 angestrebten Applikationen sind auch heute noch nicht in der Fläche verfügbar. Die Gründe dafür hat Mertens (2009, S. 29-42) detailliert beschrieben, doch stehen diese hier nicht im Vordergrund. Denn: inzwischen hat sich die IT-Welt mit serviceorientierten Architekturen, Cloud-Computing und der App-Economy bereits so weit von den 1998/2003 gegebenen organisatorischen und technischen Voraussetzungen entfernt, dass grundlegende Elemente dieser Architektur neu gedacht und neu gestaltet werden müssen.

Diese neueren Entwicklungen bilden Hintergrund und Anlass des vorliegenden Schwerpunkthefts. Insgesamt acht Beiträge wurden für das Heft in Betracht gezogen, von denen nach drei intensiven Begutachtungsrunden zwei Forschungsbeiträge zur Veröffentlichung angenommen wurden. Unser sehr herzlicher Dank gilt allen Gutachtern und Autoren, deren Engagement die hohe Qualität der nun veröffentlichten Aufsätze möglich gemacht hat. Den Herausgebern danken wir für die Möglichkeit, dieses Schwerpunkheft zu gestalten und für die professionelle Unterstützung des gesamten Prozesses.

Stand am Anfang der Gesundheitstelematik-Initiative noch die Idee - übrigens entgegen ausdrücklicher Empfehlung mehrerer renommierter Wirtschaftsinformatiker -, die in Verbindung mit der elektronischen Gesundheitskarte (eGK) geplanten Anwendungen wie elektronisches Rezept oder Notfalldaten-Management seien per Gesetz abschließend festzulegen, so könnte zukünftig der Fall eintreten, dass sich die eGK als „smart Device“ zu einer eigenständigen Plattform für vielfältige „Gesundheits-Apps“ entwickelt und als solche mit den bereits höchst erfolgreichen App-Plattformen der iOS- und Android-Welt konkurriert - genauer: konkurrieren muss. So gibt es im hoch fragmentierten Softwaremarkt des Gesundheitswesens schon heute zahlreiche Anbieter, die ihre Anwendungen im App-Store sowie auf dem Android-Market zur Verfügung stellen. Dies dürfte bereits in naher Zukunft Auswirkungen auf die Architektur bestehender Praxis- und Krankenhausinformationssysteme haben.

In ihrem Beitrag „Modulare Softwarearchitektur für Mehrwertanwendungen der deutschen Gesundheitstelematik“ befassen sich Sunyaev, Dünnebeil, Leimeister und Krcmar deshalb auf der Basis der Telematikinfrastruktur mit der Entwicklung einer Architektur, die es ermöglicht, innovative Mehrwertanwendungen zur Verbesserung der Patientenorientierung, für Qualitäts- und Effizienzsteigerungen sowie zur Verbesserung der Effektivität im digitalisierten Gesundheitswesen auf einfache Weise in bestehende Applikationen zu integrieren. Der Beitrag präsentiert, dem DesignResearch-Ansatz folgend, eine Softwarearchitektur für Mehrwertanwendungen der Telematikinfrastruktur. Die Anforderungen an die Architektur wurden aus den Anforderungen der Leistungserbringer abgeleitet. Anhand einer Mehrwertanwendung zum 
elektronischen Termin- und Überweisungsmanagement wurde sie in Kooperation mit einem großen deutschen Ärzteverbund verifiziert. Die vorgeschlagene modulare Architektur bietet damit erstmalig die Möglichkeit, beliebige Mehrwertanwendungen systematisch, einfach, sicher und zuverlässig zu entwickeln und über die zukünftige Gesundheitstelematik-Infrastruktur anzubieten und zu betreiben.

Im zweiten Beitrag untersucht Rohner mittels eines Reifegradmodells den Status des Identitätsmanagements für Behandelnde in Krankenhäusern. Obwohl dies für jedes Krankenhaus ein unverzichtbarer Bestandteil des Informationssystems sein sollte, weist das Management der Identitäten oft noch wesentliche Lücken auf. Verwechslungen oder fehlender Zugang zu Informationen in Notfallsituationen können jedoch fatale Folgen haben. Aufgrund der immer weiter zunehmenden Bedeutung von Kollaboration (Behandlungsprozesse über die Grenzen der Berufsgruppen hinweg) und Kooperation (Zusammenarbeit zwischen unterschiedlichen Leistungserbringern, bspw. Krankenhäuser, Rehabilitationseinrichtungen, Hausärzte) sind Verbesserungen jedoch unabdingbar. Der vorliegende Beitrag dokumentiert den Entwurf eines Reifegradmodells für Verantwortungs-, Organisations- und IT-Aspekte des Identitätsmanagements, zeigt einen Vorschlag für dessen Anwendung und berichtet im Sinne der Design-ResearchEvaluation über Piloteinsätze in zwei großen Schweizer Krankenhäusern. - Nur am Rande sei hier darauf hingewiesen, dass die im Gesundheitswesen schon heute vorhandenen Lösungen für ein ausgefeiltes, leistungsfähiges Identitätsmanagement im Sinne der Konvergenz im heraufziehenden Internet der Dinge auch für andere Branchen von hohem Interesse sein dürfte.

Mit Fragen des Innovationsmanagements, der Innovations- und Diffusionsprozesse sowie der spezifischen Innovationshürden im Gesundheitswesen befasst sich Herr Dr. v. Baehr, Geschäftsführer der Bosch Healthcare GmbH, im Interview des Schwerpunkthefts. Dabei tritt deutlich zutage, dass die im internationalen Vergleich sehr große Zahl machtvoller Anspruchsgruppen im bundesdeutschen Gesundheitswesen selbst für die Einführung moderner digitaler Lösungen hohe Hürden aufbauen kann und aufbaut. Allerdings, auch das wird im Interview diskutiert, könnten gerade die jüngsten Entwicklungen in der Softwareindustrie - bspw. Serviceorientierung, Cloud-Computing und App-Economy - im Verein mit dem Internet der Dinge und dem Programm Industrie 4.0 der Bundesregierung wichtige Innovationsschübe auslösen, deren Trag- und Reichweite heute noch nicht in vollem Umfang abzusehen ist. - Es bleibt spannend!

Ihnen, den Leserinnen und Lesern, wünschen wir nun Freude und Gewinn beim Studium der Beiträge: das Gesundheitswesen bietet gerade den Wissenschaftlern und Praktikern der Wirtschaftsinformatik zahlreiche interessante Herausforderungen. Einige Wesentliche davon finden Sie in diesem Heft!

\section{Literatur}

Mertens P (2009) Fehlschläge bei IT-Großprojekten der Öffentlichen Verwaltung, 3. Aufl. Universität Erlangen-Nürnberg, Arbeitspapier Nr. 1/2009

Roland Berger \& Partner (1998) Telematik im Gesundheitswesen - Perspektiven der Telemedizin in Deutschland. Bundesministerium für Bildung, Wissenschaft, Forschung und Technologie in Zusammenarbeit mit dem Bundesministerium für Gesundheit, München

ZTG-Telematik-Glossar (oJ) better IT for better health (Abk.: bIT4health). http://www. egesundheit.nrw.de/content/ztg_telematik_glossar/index_ger.html?selected=B. Abruf am 2012-10-21 